\title{
Capacidade de combinação para oito caracteres agronômicos em culti- vares de feijão-de-vagem de crescimento determinado ${ }^{1}$.
}

\author{
Ana Cristina P. P. de Carvalho ${ }^{2}$, Nilton R. Leal ${ }^{3}$, Rosana Rodrigues ${ }^{3}$, Francisco Ademar Costa ${ }^{4}$ \\ ${ }^{2}$ PESAGRO-RIO (Estação Experimental de Itaguaí), Rodovia Rio - São Paulo Km 47, 23851-970 Seropédica - RJ; ${ }^{3}$ UENF, Av. Alberto \\ Lamego 2000, 28015-620, Campos dos Goytacazes - RJ; ${ }^{4}$ UFRRJ - Depto. de Matemática, Rodovia Rio - São Paulo Km 47, $23851-970$ \\ Seropédica - RJ.
}

\section{RESUMO}

Para estudar os efeitos da hibridação entre cultivares rasteiras de feijão-de-vagem, foi conduzido um experimento na área de olericultura da Estação Experimental de Itaguaí da PESAGRO-RIO. As cultivares Alessa, Andra, Cota e Cascade foram utilizadas como progenitores e cruzadas em esquema de dialelo sem os recíprocos. As cultivares parentais e os híbridos $\mathrm{F}_{1}$ foram cultivados em condições de campo, sendo avaliados oito caracteres agronômicos. Efeitos significativos para capacidade geral de combinação (CGC) foram identificados para os caracteres número de vagens, peso de vagens, comprimento e diâmetro das vagens, número de dias para o início e o término do florescimento e altura de plantas. Para capacidade específica de combinação (CEC), houve significância para todos os caracteres, indicando que efeitos de dominância e/ou epistáticos estão envolvidos no controle das características. Para o conjunto dos caracteres testados, a cultivar Cota foi identificada como o melhor progenitor. Em termos de produção, duas combinações, 'Alessa' x 'Cascade' e 'Cota' $x$ 'Cascade' apresentaram resultados promissores.

Palavras-chave: Phaseolus vulgaris L., dialelo, produção de vagens, capacidade geral de combinação, capacidade específica de combinação.

\begin{abstract}
Combining ability of eight agronomic characters in bush snap bean cultivars.

In order to study the effects of hybridisation on bush snap bean genotypes, a test was conducted at PESAGRO-RIO. Four bush snap bean cultivars, Alessa, Andra, Cota and Cascade were used as parents and crossed in a diallel mating design without reciprocals. Parental cultivars and $F_{1}$ hybrids were planted under field conditions and eight agronomic characters were evaluated. Significant effects for general combining ability (GCA) were identified for pod number, pod weight, pod length, pod diameter, days from planting date to flowering, and plant height. For specific combining ability (SCA), there was significance for all characters, showing that dominance/ epistatic effects were involved in control of characteristics. For all traits evaluated, 'Cota' was considered the best parental. For yield characters, two hybrids, 'Alessa' x 'Cascade' and 'Cota' x 'Cascade' showed the best results.
\end{abstract}

Keywords: Phaseolus vulgaris L., diallel crosses, pod yield, general combining ability, specific combining ability.

\section{(Aceito para publicação em 08 de março de 1999)}

$\mathrm{O}$ feijão-de-vagem (Phaseolus vulgaris L.) é cultivado na maioria dos estados brasileiros, com destaque para São Paulo, Rio de Janeiro e Minas Gerais. No Brasil, a cultura é basicamente destinada à produção de vagens verdes para o comércio do produto "in natura" (Leal, 1987b). As principais cultivares tradicionalmente utilizadas apresentam crescimento indeterminado, com vagens achatadas, semi-achatadas ou arredondadas (Leal, 1987b; Castellane et al.,1988). A introdução de cultivares importadas, de hábito de crescimento determinado, com vagens do tipo cilíndrico, tem sido tentada, uma vez que a cultura rasteira permite mecanizar as operações culturais, inclusive a colheita (Filgueira, 1981).
Além disso, plantas desse tipo são vigorosas, eretas, com vagens de $15 \mathrm{~cm}$ de comprimento, muito tenras e sem fibras.

Na década de 80, cultivares de hábito de crescimento determinado, como a Cascade (Barnes, 1970; Leal, 1983), foram introduzidas de outros países, e obtidas no Brasil através de programas de melhoramento genético, como por exemplo, Alessa (Leal, 1990a), Andra (Leal,1990b), Mimoso Rasteiro AG-461 (Carrijo, 1991) e Cota (Leal, 1987a). Essas cultivares têm contribuído para a expansão da cultura em diversas regiões a um custo operacional mais baixo, produzindo vagens de boa qualidade (Leal, 1987b). Entre as vantagens da utilização de cultivares rasteiras, podem ser citadas a redução acentuada do período de produção e do número de colheitas; eliminação do tutoramento; diminuição no uso de defensivos; melhor aproveitamento e proteção do solo; menor tempo de utilização da área cultivada; elevada produção; economia de mão-de-obra e insumos em geral, e boa aceitação do produto colhido (Leal et al., 1983).

O objetivo de grande parte dos programas de melhoramento é aumentar e estabilizar o rendimento a um custo de produção que permita aos produtores uma maximização do retorno econômico (Zimmermann et al., 1996).

Pelo uso do método dialélico é possível a determinação de estimativas de parâmetros importantes para a seleção de progenitores e dos efeitos genéticos

\footnotetext{
${ }^{1}$ Parte da dissertação de Mestrado em Genética apresentada pelo primeiro autor à Universidade Federal do Rio de Janeiro.
} 
envolvidos no controle dos caracteres estudados (Ramalho et al., 1993). Na metodologia de análise dialélica proposta por Griffing (1956), o desempenho médio de cada genótipo é decomposto em capacidade geral de combinação (efeitos principais) e capacidade específica de combinação (interações). A capacidade geral de combinação (CGC) diz respeito ao comportamento médio de um progenitor numa série de combinações híbridas e está associada aos efeitos aditivos dos alelos, e às ações epistáticas do tipo aditiva (Cruz \& Vencovsky, 1989). Por sua vez, a capacidade específica de combinação (CEC) é usada para estimar os desvios do comportamento de um híbrido em relação ao esperado com base na CGC, estando associada aos efeitos dos desvios de dominância e epistasia envolvendo dominância (Cruz \& Vencovsky, 1989; Cruz \& Regazzi, 1994).

Alguns estudos genéticos têm sido realizados com o feijão-de-vagem, como base para o melhoramento da cultura, envolvendo cruzamentos dialélicos (Dickson, 1967; Hamad, 1975; Park \& Davis, 1976; Leal, 1978; Singh \& Saini, 1982, e Leal et al., 1982).

Os objetivos do presente trabalho foram estimar os efeitos genéticos envolvidos no controle de oito características do feijão-de-vagem, utilizando a metodologia de cruzamentos dialélicos, entre quatro cultivares.

\section{MATERIAL E MÉTODOS}

Quatro cultivares de feijão-de-vagem de hábito determinado (Alessa, Andra, Cota e Cascade) foram utilizadas para a produção de híbridos em esquema dialélico $4 \times 4$, sem os recíprocos. Utilizou-se a metodologia de hibridação descrita por Antunes et al. (1980), na qual não se utiliza nenhuma proteção no estigma após a emasculação e polinização. Os cruzamentos foram efetuados em condições de casa-de-vegetação na Estação Experimental de Itaguaí (EEI) da PESAGRO-RIO. Os seis híbridos $\mathrm{F}_{1}$ e os quatro progenitores foram avaliados em condições de campo na EEI, no período de maio a agosto de 1990. O delineamento experimental utilizado foi blocos casualizados, com cinco repetições. Cada

Tabela 1. Estimativas dos efeitos da Capacidade Geral de Combinação para oito caracteres avaliados em cultivares e híbridos $\mathrm{F}_{1}$ de feijão-de-vagem (Phaseolus vulgaris L.). Itaguaí (RJ), PESAGRO-RIO, 1990.

\begin{tabular}{|c|c|c|c|c|}
\hline \multirow{2}{*}{ CARACTERES } & \multicolumn{4}{|c|}{ EFEITOS(gi) } \\
\hline & Alessa & Andra & Cota & Cascade \\
\hline NTV $^{1}$ & $-3,57 * \star$ & $-15,40 * *$ & $10,57 * *$ & $8,40 * *$ \\
\hline $\mathrm{PTV}^{2}$ & $-50,60 * *$ & $-73,00 * *$ & $93,63^{* *}$ & $29,97 * \star$ \\
\hline $\mathrm{CVA}^{3}$ & $0,04 * *$ & $-0,11 * *$ & $0,49 * *$ & $-0,43 * *$ \\
\hline DVA $^{4}$ & $-0,06 * *$ & 0,06 ** & $-0,05^{\star *}$ & $0,05^{* *}$ \\
\hline $\mathrm{NIF}^{5}$ & $-0,05^{\star \star}$ & $0,07 * *$ & $-0,62^{* *}$ & $-0,02 * *$ \\
\hline $\mathrm{NFF}^{6}$ & $-0,07^{\star}$ & $0,53^{*}$ & $-0,60 *$ & $0,13^{*}$ \\
\hline $\mathrm{FIB}^{7}$ & $-0,01$ & $-0,01$ & 0,03 & $-0,01$ \\
\hline $\mathrm{ALT}^{8}$ & $-0,004 * *$ & $-0,601 * *$ & 1,594 ** & $-0,988 * *$ \\
\hline
\end{tabular}

**, * Significativo ao nível de 1 e $5 \%$, respectivamente, pelo teste de Tukey.

${ }^{1} \mathrm{NTV}=$ Número total de vagens; ${ }^{2} \mathrm{PTV}=$ Peso total de vagens; ${ }^{3} \mathrm{CVA}=$ Comprimento da vagem $;{ }^{4} \mathrm{DVA}=$ Diâmetro da vagem $;{ }^{5} \mathrm{NIF}=$ Número de dias para o início de florescimento; ${ }^{6} \mathrm{NFF}=$ Número de dias para o fim do florescimento $;{ }^{7} \mathrm{FIB}=$ Teor de fibra da vagem; ${ }^{8}$ ALT $=$ Altura das plantas.

parcela foi composta por dez plantas distribuídas em duas linhas, espaçadas de $0,40 \mathrm{~m}$, com 0,20 m entre plantas. Foram feitos os tratos culturais normais recomendados para a cultura (Filgueira, 1981; Castellane et al., 1988). As plantas foram conduzidas sem estaqueamento e irrigadas por infiltração. As seguintes características foram avaliadas: número total de vagens; peso total de vagens; comprimento médio e diâmetro médio das vagens; número de dias para o início do florescimento; número de dias para o fim do florescimento; teor de fibra das vagens e altura das plantas.

O teor de fibra nas vagens foi determinado em $100 \mathrm{~g}$ de vagens sem sementes, de acordo com a metodologia utilizada por Kramer (1951), citado por Corrêa (1985). Este método consiste em triturar as vagens por cinco minutos, num liqüidificador com $200 \mathrm{ml}$ de água, seguindo-se a transferência do material triturado para uma peneira de metal de 30 mesh, previamente pesada, lavagem em água corrente e secagem em estufa a $100^{\circ} \mathrm{C}$ por duas horas. Em seguida, o material deve ser pesado, subtraindose o peso da peneira.

As análises de capacidade combinatória foram realizadas de acordo com o Método 2, no qual são incluídas as cultivares parentais e o conjunto de híbridos $\mathrm{F}_{1}$, num total de $\mathbf{p}(\mathbf{p}+\mathbf{1}) / \mathbf{2}$ genótipos, considerando-se o Modelo 1, em que se supõe que as cultivares são de efeito fixo e apenas o erro experimental é de efeito aleatório (Ramalho et al., 1993). Nesse Modelo, todas as conclusões devem, ser limitadas aos genótipos em estudo no presente trabalho.

O modelo estatístico adotado analisa a tabela dialélica com base na média das repetições, decompondo-a nos seguintes efeitos:

$Y_{i j}=m+g_{i}+g_{j}+s_{i j}+e_{i j}$, em que:

$\mathrm{Y}_{\mathrm{ij}}=$ valor médio da combinação híbrida ( $\mathrm{i}^{1} \mathrm{j}$ ) ou do progenitor $(\mathrm{i}=\mathrm{j}) ; \mathrm{m}$ = efeito médio de todos os tratamentos; $\mathrm{g}_{\mathrm{i}}=$ efeito da CGC do progenitor $\mathrm{i}$, e corresponde ao desvio de seu desempenho médio em combinações híbridas; $\mathrm{g}_{\mathrm{j}}$ $=$ efeito da CGC do progenitor $\mathrm{j} ; \mathrm{s}_{\mathrm{ij}}=$ efeito da CEC para os cruzamentos entre os progenitores i e j, sendo $\mathrm{s}_{\mathrm{ij}}=\mathrm{s}_{\mathrm{ji}}$; e $\mathrm{e}_{\mathrm{ij}}=$ erro experimental médio associado às médias da tabela dialélica.

\section{RESULTADOS E DISCUSSÃO}

Houve diferença significativa entre os genótipos avaliados, permitindo a decomposição dos efeitos de tratamentos em capacidade geral e específica de combinação (CGC e CEC).

Para a CGC houve diferença significativa para os caracteres número e peso total de vagens, comprimento e diâmetro das vagens, número de dias para o início e fim do florescimento e altura das plantas. Não foi detectada diferença na CGC para o caráter teor de fibra das vagens (Tabela 1). 
Tabela 2. Estimativas dos efeitos da Capacidade Específica de Combinação para oito caracteres avaliados em cultivares e híbridos $\mathrm{F}_{1}$ de feijão-de-vagem (Phaseolus vulgaris L.). Itaguaí (RJ), PESAGRO-RIO, 1990.

\begin{tabular}{|c|c|c|c|c|c|c|}
\hline \multirow{2}{*}{ CARACTERES } & \multicolumn{6}{|c|}{ EFEITOS (SIJ) } \\
\hline & $1 \times 2$ & $1 \times 3$ & $1 \times 4$ & $2 \times 3$ & $2 \times 4$ & $3 \times 4$ \\
\hline NTV & $1,527^{* *}$ & $-20,240^{* *}$ & $34,127^{* *}$ & $-14,207^{* *}$ & $-32,440 * *$ & $74,793^{* *}$ \\
\hline PTV & $51,920 * *$ & $-96,513^{* *}$ & $77,553^{* *}$ & $-65,313^{\star *}$ & $-224,447^{\star *}$ & 530,120 ** \\
\hline CVA & $0,627^{* *}$ & $0,227^{* *}$ & $-0,363^{* *}$ & $-0,229 * *$ & $0,461^{* *}$ & 1,281 ** \\
\hline DVA & $0,010^{* *}$ & $0,011 * *$ & $0,013^{* *}$ & $0,002^{* *}$ & $0,035^{* *}$ & $-0,055^{\star *}$ \\
\hline NIF & $-0,213^{* *}$ & $1,286 * *$ & $0,686^{* *}$ & $0,953 * *$ & $3,953^{* *}$ & $-1,146 * *$ \\
\hline NFF & $-0,227^{* *}$ & $1,307^{* *}$ & $0,573^{* *}$ & $1,107^{* *}$ & $3,173^{* *}$ & $-1,293^{* *}$ \\
\hline FIB & $-0,167^{* *}$ & $0,203^{* *}$ & 0,070 ** & $-0,150 * *$ & $0,117^{* *}$ & $-0,053^{* *}$ \\
\hline ALT & $1,765^{\star *}$ & $-0,652 * *$ & $-0,969 * *$ & $-0,195 * *$ & $-1,652 * *$ & $2,351 * *$ \\
\hline
\end{tabular}

** Significativo ao nível de $1 \%$ de probabilidade pelo teste de Tukey.

${ }^{\mathrm{x} /}{ }^{1} \mathrm{NTV}=$ Número total de vagens $;{ }^{2} \mathrm{PTV}=$ Peso total de vagens $;{ }^{3} \mathrm{CVA}=$ Comprimento da vagem $;{ }^{4} \mathrm{DVA}=$ Diâmetro da vagem $;{ }^{5} \mathrm{NIF}=$ Número de dias para o início de florescimento $;{ }^{6} \mathrm{NFF}=$ Número de dias para o fim do florescimento $;{ }^{7} \mathrm{FIB}=$ Teor de fibra da vagem; ${ }^{8} \mathrm{ALT}$ $=$ Altura das plantas.

(1) Alessa, (2) Andra, (3) Cota, (4) Cascade

Significância para CGC indica que efeitos gênicos aditivos estão envolvidos no controle genético da característica. Os efeitos gênicos aditivos são fixados ao longo de sucessivas gerações, sendo importantes sobretudo para espécies autógamas como o feijão-de-vagem, nas quais o uso de híbridos não é economicamente viável. A existência de efeitos gênicos aditivos para todos os caracteres avaliados permite supor a possibilidade de se obter novas cultivares derivadas de populações segregantes a partir de cruzamentos com os progenitores testados.

Estimativas correspondendo a altos valores de $\mathrm{g}_{\mathrm{i}}$, positivos ou negativos, indicam progenitores muito superiores ou inferiores aos demais progenitores do dialelo em relação à média dos cruzamentos. Os valores de CGC são indicadores da importância de genes de efeitos predominantemente aditivos e progenitores que apresentem as maiores CGC devem ser potencialmente superiores e podem ser incluídos em programas de melhoramento visando seleção de novas linhagens puras em gerações avançadas (Ramalho et al., 1993).

Pelos valores de $\mathrm{g}_{\mathrm{i}}$ obtidos para produção em número e peso de vagens, 'Cota' foi identificada como o melhor progenitor, seguida da cultivar Cascade. Para o feijão-de-vagem, estudos sobre o controle genético da produção de vagens têm revelado um controle poligênico. Em análise dialélica de caracteres de importância em vagens, resultados apresentados por Dickson (1967), mostraram que houve predomínio da ação aditiva para o número de vagens por planta e número de sementes por planta.

Em termos de comprimento de vagem, as cultivares Andra e Cascade, apresentaram valores negativos para CGC, indicando que contribuíram para reduzir o comprimento das vagens nos cruzamentos em que participaram. 'Alessa' e 'Cota', por sua vez, contribuíram para aumentar o comprimento das vagens. Para o comprimento da vagem, o trabalho de Dickson (1967) mostrou que houve significância apenas para o efeito aditivo.

Para o diâmetro das vagens, 'Alessa' e 'Cota', ambas apresentando vagens do tipo chata, obtiveram valores negativos de $\mathrm{g}_{\mathrm{i}}$, demonstrando que atuaram reduzindo o diâmetro nos cruzamentos em que estiveram envolvidas. 'Andra' e 'Cascade', que produzem vagens do tipo redondo, ao contrário, atuaram no sentido de aumentar o diâmetro das vagens.

Com relação ao ciclo de cultivo, 'Alessa' e 'Cota' influenciaram nos cruzamentos diminuindo o número de dias para o início e o fim do florescimento, enquanto 'Andra' contribuiu aumentando tanto o número de dias para o início quanto para o fim do florescimento. A cultivar Cascade apresentou comporta- mento diferenciado para as duas características, diminuindo o número de dias para o início do florescimento, porém, retardando o fim do mesmo.

Apenas a cultivar Cota apresentou valores positivos para $\mathrm{g}_{\mathrm{i}}$, indicando que foi a única que contribuiu para aumentar a altura das plantas.

Deve-se ressaltar que as estimativas $\mathrm{g}_{\mathrm{i}}$ são válidas apenas para o conjunto de progenitores testados nesse estudo (Vencovsky \& Barriga, 1992).

Os efeitos de capacidade específica de combinação $\left(\mathrm{s}_{\mathrm{ij}}\right)$ foram significativos para todos os caracteres avaliados (Tabela 2). Os efeitos da CEC (sij) são medidas dos efeitos gênicos nãoaditivos e dependem de genes que mostrem efeitos de dominância e/ou epistasia (Cruz \& Regazzi, 1994). Para $\mathrm{s}_{\mathrm{ij}}$, valores de pequena magnitude são indicadores de que os híbridos $F_{1}$ entre os parentais em questão, apresentaram comportamento esperado com base na CGC dos progenitores. Por outro lado, altos valores absolutos de $\mathrm{s}_{\mathrm{ij}}$ indicam que o desempenho de um híbrido em particular é melhor ou pior em relação ao esperado com base na CGC dos progenitores.

Valores de $s_{i j}$ negativos foram registrados para as combinações 'Alessa' x 'Cota', 'Andra' x 'Cota' e 'Andra' x 'Cascade' em termos de produção de número e peso de vagens. As demais combinações obtiveram valores 
positivos para $\mathrm{s}_{\mathrm{ij}}$, com o híbrido 'Cota' $\mathrm{x}$ 'Cascade' apresentando os maiores valores.

Considerando todos os caracteres, a combinação 'Cota' x 'Cascade' foi a que apresentou resultados mais promissores. Em termos de produção, destacou-se também a combinação 'Alessa' $x$ 'Cascade' (Tabela 2).

A obtenção de gerações avançadas a partir dos híbridos promissores permitirá a continuidade dos estudos genéticos dos caracteres aqui apresentados, bem como a seleção de progênies superiores que poderão resultar em novas cultivares rasteiras de feijão-de-vagem.

\section{LITERATURA CITADA}

ANTUNES, I. F.; TEIXEIRA, M. G. ; ZIMMERMANN, M. J. O. Relative performance among crossing techniques under field and greenhouse conditions on beans. Annual Report Bean Improvement Cooperative, n. 23, p. 117 - 119. 1980.

BARNES, W. C. New vegetable varieties list XVII. Hortscience, v. 5, n. 3, p. 146 - 149. 1970.

CARRIJO, I. 'Mimoso Rasteiro AG-461': Nova cultivar de feijão-de-vagem. Horticultura Brasileira, Brasília n. 9, v. 2, p. 96. 1991.

CASTELLANE, P. D.; VIEIRA, R. D.; CARVALHO, N. M. de. Feijão-de-vagem (Phaseolus vulgaris L.): cultivo e produção de sementes. FUNEP/FCAV-UNESP, Jaboticabal, 60 p., 1988

CORRÊA, T. B. S. Avaliação tecnológica de cultivares de feijão-de-vagem (Phaseolus vulgaris L.) para industrialização. Itaguaí, RJ, Universidade Federal Rural do Rio de Janeiro, 1985. 183 p. (Tese mestrado).
CRUZ, C. D.; REGAZZI, A. J. Modelos biométricos aplicados ao melhoramento genético. Viçosa: Imprensa Universitária. 1994. $390 \mathrm{p}$.

CRUZ, C. D.; VENCOVSKY, R. Comparação de alguns métodos de análise dialélica. Revista Brasileira de Genética, Ribeirão Preto, v. 12 , n. 2, p. 425 - 438, 1989.

DICKSON, M. H. Diallel analysis of seven economic characters in snap bean. Crop Science, v. 7, n. 3, p. 121 - 124, 1967.

FILGUEIRA, F. A. R. Manual de olericultura: cultura e comercialização de hortaliças. 2 ed. São Paulo. Ed. Agronômica Ceres. v. 1, 1981. $338 \mathrm{p}$.

GRIFFING, B. Concept of general and specific combining ability in relation to diallel crossing systems. Australian Journal of Biological Science, v. 9, p. 463 - 493, 1956.

HAMAD, I. A. Inheritance of yield, yield components, number of days to flowering, plant height and incidence of intelocular cavitation of pods in snap beans (Phaseolus vulgaris L.). Madison, EUA, University of Wisconsin, 1975. 118 p. (Tese doutorado).

LEAL, N. R. Alessa - Nova cultivar de feijão-devagem. Horticultura Brasileira, Brasília, v. 8, n. 1, p. 29 - 30, 1990a.

LEAL, N. R. Andra - Nova cultivar de feijão-devagem. Horticultura Brasileira, Brasília, v. 8 , n. 1, p. 29, 1990 b.

LEAL, N. R. Combining ability analysis and evaluation of near-homozygous lines of snap bean (Phaseolus vulgaris L.). Madison, EUA University of Wisconsin. 1978. 77 p. (Tese doutorado).

LEAL, N. R. Cota - Nova cultivar de feijão-devagem de porte determinado. Horticultura Brasileira, Brasília, v. 5, n. 1, p. 62, 1987a.

LEAL, N. R. Produção e melhoramento de feijão-de-vagem (Phaseolus vulgaris L.) no Brasil. In: El mejoramineto genético de la habichuela en America Latina; memorias de un taller. Cali, Colômbia, CIAT, p. 135 - 147. (Documento de trabajo, 30), 1987b.
LEAL, N. R.; ARAÚJO, M. L. de; LIBERAL, M. T.; CRUZ JÚNIOR, F. G. da. Avaliação comparativa entre cultivares estaqueadas e rasteiras de feijão-de-vagem. In: Congresso Brasileiro de Olericultura, 23, Rio de Janeiro, 1983. Resumos. Rio de Janeiro, SOB, p. 42.

LEAL, N. R.; HAMAD, I. A.; BLISS, F. A. Avaliação de progenitores e linhas avançadas de melhoramento de feijão-de-vagem de crescimento determinado. Pesquisa Agropecuária Brasileira, Brasília, v. 17, n. 2, p. 225 - 231, 1982.

PARK, H. G.; DAVIS, D. W. Inheritance of interlocular cavitation in a six-parent diallel cross in snap bean (Phaseolus vulgaris L.). Journal of the American Society for Horticultural Science, v. 10, n. 2, p. 184 - 189, 1976.

RAMALHO, M. A. P.; SANTOS, J. B. dos; ZIMMERMANN, M. J. de O. Genética quantitativa em plantas autógamas; aplicações ao melhoramento do feijoeiro. Goiânia: Editora da UFG, 1993. 271 p.

VENCOVSKY, R.; BARRIGA, P. Genética biométrica no fitomelhoramento. Ribeirão Preto: Sociedade Brasileira de Genética, 1992. 469 p.

SINGH, A. K.; SAINI, S. S. A. A note on combinig ability in French bean (Phaseolus vulgaris L.). Haryana Journal of Horticultural Science, v. 11, n. 3 - 4, p. 270 - 273, 1982.

ZIMMERMANN, M. J. de O.; CARNEIRO, J. E. S.; PELOSO, M. J. Del; COSTA, J. G. C.; RAVA, C. A.; SARTORATO, A.; PEREIRA, P. A. A. Melhoramento genético e cultivares. In; Araújo, R. S., Rava, C. A., Stone, L. F., Zimmermman, M. J. de O. (coords.), Cultura do feijoeiro comum no Brasil. Piracicaba: Associação Brasileira para Pesquisa da Potassa e do Fosfato, 1996. p. 223 - 273. 